\title{
Fluorescent Nanodiamonds Encapsulated by Cowpea Chlorotic Mottle Virus (CCMV) Proteins for Intracellular 3D-Trajectory Analysis
}

Yingke Wu $\%$ Shuqin Cao $\%$ Md Noor A Alam, Marco Raabe, Sandra Michel-Souzy, Zuyuan Wang, Manfred Wagner, Anna Ermakova, Jeroen J.L.M. Cornelissen*, Tanja Weil*

Y. Wu, M. N. A. Alam, Dr. M. Raabe, Dr. Z. Wang, Dr. A. Ermakova, Dr. M. Wagner, Prof. Dr. T. Weil

Max Planck Institute for Polymer Research, Ackermannweg 10, 55128 Mainz, Germany

*E-mail: weil@mpip-mainz.mpg.de

Dr. A. Ermakova

Institute for Physics, Johannes Gutenberg University Mainz, Staudingerweg 7, 55128 Mainz, Germany

Dr. S. Cao, Dr. S. Michel-Souzy, Prof. Dr. J. J.L.M. Cornelissen

Laboratory for Biomolecular Nanotechnology, MESA+ Institute for Nanotechnology, University of Twente, P.O. Box 217, 7500 AE, Enschede, The Netherlands

*E-mail: j.j.1.m.cornelissen@utwente.nl

M. N. A. Alam, Dr. M. Raabe, Prof. Dr. T. Weil

Institute of Inorganic Chemistry I, Ulm University, Albert-Einstein-Allee 11, 89081 Ulm, Germany

Dr. S. Cao

State Key Laboratory of Oral Diseases, West China Hospital of Stomatology, Sichuan University, Chengdu 610041, China

Dr. Z. Wang 
Institute for Measurement and Automation, Division of Sensor Technology and Measurement Systems, Bundeswehr University Munich, Werner-Heisenberg-Weg 39, 85579 Neubiberg, Germany

Keywords: Nanodiamond, virus capsid protein, intracellular trajectory, diffusion coefficient, mean square displacement.

Abstract Long-term tracking of nanoparticles to resolve intracellular structures and motions is essential to elucidate fundamental parameters as well as transport processes within living cells. Fluorescent nanodiamond (ND) emitters provide cell compatibility and very high photostability. However, high stability, biocompatibility, and cellular uptake of these fluorescent NDs under physiological conditions are required for intracellular applications. Herein, highly stable NDs encapsulated with Cowpea chlorotic mottle virus capsid proteins (ND-CP) are prepared. A thin capsid protein layer is obtained around the NDs, which imparts reactive groups and high colloidal stability, while retaining the optomagnetic properties of the coated NDs as well as the secondary structure of CPs adsorbed on the surface of NDs. In addition, the ND-CP shows excellent biocompatibility both in vitro and in vivo. Long-term 3D trajectories of the ND-CP with fine spatiotemporal resolutions are recorded; their intracellular motions are analyzed by different models, and the diffusion coefficient are calculated. The ND-CP with its brilliant optical properties and stability under physiological conditions provides us with a new tool to advance the understanding of cell biology, e.g., endocytosis, exocytosis, and active transport processes in living cells as well as intracellular dynamic parameters. 
Nanodiamonds (NDs), which consist of an all-carbon $\mathrm{sp}^{3}$-scaffold, exhibit both exciting opto-magnetic properties and excellent biocompatibility. Point defects in the lattice of NDs, such as nitrogen-vacancy (NV) centers, provide stable fluorescence without bleaching or blinking, even after several months of continuous irradiation. ${ }^{1}$ In addition, the emission wavelength of NDs is size-independent and tunable from the visible to the near-infrared region by using different defects (e.g. $\mathrm{SiV}, \mathrm{GeV}$ etc.). ${ }^{2}$ Hence, NDs are widely used in bioimaging $^{3-5}$, and drug delivery ${ }^{6-10}$. In addition, it has been proven that NDs can act as cellular biomarkers for long-term intracellular tracking. ${ }^{11-13}$ Furthermore, NDs containing negatively charged NV centers can also serve as single-spin sensors to detect many essential properties in the biological microenvironment, such as temperature ${ }^{14-16}$, magnetic fields ${ }^{17,18}$, electron spins ${ }^{19,20}$, and strain ${ }^{21}$ with nanoscale resolution.

For most of these applications, appropriate surface functionalization of NDs is required because the poor colloidal stability of unmodified NDs under physiological conditions leads to uncontrolled aggregation in aqueous media ${ }^{22}$. Surface functionalization, like harsh chemical treatments or air oxidation, improves surface uniformity and provides new binding sites for the attachment of drug molecules, dyes, targeting groups, or antibodies. ${ }^{23}$, ${ }^{24}$ Furthermore, surface coating is crucial to avoid foreign body interactions of the particles in vivo ${ }^{25}$ and allows the nanoparticles to accumulate and remain at the target sites for a 
prolonged time period. Different surface coatings have been developed for this purpose, such as silica ${ }^{26}$, hyperbranched polyglycerol $(\mathrm{HPG})^{27}, \operatorname{poly}(\mathrm{L}-\mathrm{DOPA})^{7}$, insulin $^{28}$, and human serum albumin (HSA), which was modified with polymers ${ }^{6}$, among others. In addition to synthetic common protein coatings, hybrid virus-like particles have recently gained attention due to their straightforward preparation, high biocompatibility, and unique properties and their application as drug delivery vehicles. ${ }^{29}$

Viruses are evolutionary optimized carrier systems. Uptake and diffusion pathways of viruses to their host cells are still barely understood. ${ }^{30}$ However, it is very important to elucidate virus cell interactions and their trafficking inside cells in order to identify new therapeutic interventions or to mimic such processes for improved drug delivery of viruslike nanoparticle transporters. Cowpea chlorotic mottle virus (CCMV) has gained great interest in recent years as tools in nanotechnology as well as for the development of targeted drug delivery vehicles due to their perfectly defined structure, high stability, good biocompatibility, homogeneity, self-assembly, and low toxicity. ${ }^{31}$ The size of the native CCMV is about $30 \mathrm{~nm}$ in diameter and can be disassembled into capsid proteins (CP). The genome can be removed by centrifugation under high salt concentrations at neutral $\mathrm{pH}^{32}$ The thus obtained $\mathrm{CPs}$ reassemble into either empty capsids at lower $\mathrm{pH}$ or virus-like particles (VLPs) at neutral $\mathrm{pH}$ using templates such as negatively charged inorganic nanoparticles, ${ }^{33}, 34$ negatively charged polymers, ${ }^{35}$ enzymes, ${ }^{36}$ and organic 
aggregates ${ }^{37}$ among others. Furthermore, by controlling the $\mathrm{pH}$ and ionic strength of the assembly buffer, CCMV can be reassembled into a variety of geometries such as tubes, multilayered structures, and dumbbells. ${ }^{38-41}$ Despite their broad applications, CCMV cell uptake and diffusion inside cells is still barely understood.

Here, we report the preparation and characterization of fluorescent nanodiamonds encapsulated within cowpea chlorotic mottle virus capsid proteins (ND-CP) as model system for fluorescence imaging and single particle tracking applications inside living cells. We believe that our strategy could also be applicable to other viruses, which could give new insights into their intracellular pathways, which would be of fundamental interest as well as for the design of improved drug delivery systems.

A schematic outline of the preparation of ND-CP is displayed in Figure 1. Firstly, CPs were isolated from the $C C M V$ and stored in a capsid storage buffer according to our previous work $^{34}$. Before encapsulation of the NDs, the CPs were transferred into a coat protein buffer. Then NDs in a polyvinylpyrrolidone (PVP) solution were added to the CPs and the mixture was stirred at $4{ }^{\circ} \mathrm{C}$ overnight. 


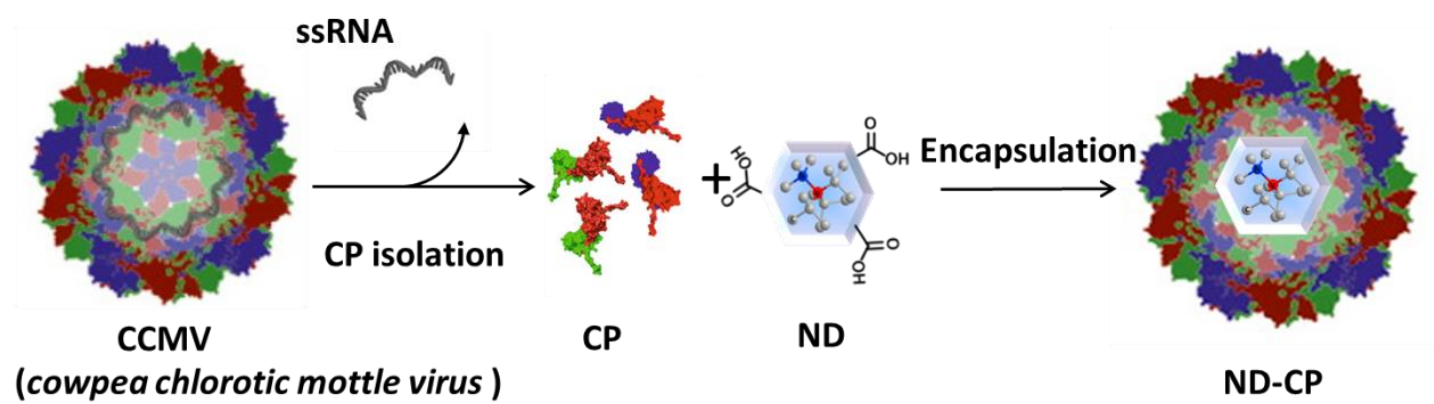

Figure 1. Schematic illustration of the preparation of cowpea chlorotic mottle virus capsid protein encapsulated fluorescent nanodiamonds (ND-CP).

Free CPs and encapsulated ND-CPSs were separated and removed by fast protein liquid chromatography (FPLC) equipped with a UV-vis detector. As shown in Figure 2A, the NDs coated with CP both in PBS buffer and Tris buffer eluted at a lower volume $(V \approx 8 \mathrm{~mL})$ than the native CCMV virus $(V \approx 9.5 \mathrm{~mL})^{33}$, suggesting that NDs were successfully coated. The successful coating of NDs with CPs was also confirmed by sodium dodecyl sulfate-polyacrylamide gel electrophoresis (SDS-PAGE) (Figure S2), which shows that the band of purified ND-CP appeared at the same position as native CCMV. To test whether the CPs retained their native structure after adsorption to the ND surface, the secondary structure was investigated by circular dichroism (CD) spectroscopy. As shown in Figure 2B, only native $\mathrm{CP}$ and ND-CP showed a signal in $\mathrm{CD}$, and both spectra were very similar, indicating that the native structure of $\mathrm{CP}$ was not affected. Subsequently, the hydrodynamic sizes of the ND-CP were characterized by dynamic light scattering (DLS) (Figure 2C). The average hydrodynamic diameters changed from $35 \pm 2 \mathrm{~nm}$ in water before coating to $46 \pm$ 
$3 \mathrm{~nm}$ for NDs in Tris buffer containing PVP, and $52 \pm 5 \mathrm{~nm}$ for ND-CP in Tris buffer, respectively. Furthermore, ND-CP showed a monomodal distribution in DLS. Uncoated NDs and ND-CP were characterized in terms of their distribution, shape, and morphology by transmission electron microscopy (TEM). Whereas bare NDs were prone to significant aggregation and displayed a heterogeneous distribution (Figure 2D), non-aggregating, homogeneous single particles were observed for ND-CP (Figure 2E). The histogram analysis of TEM images of ND and ND-CP showed a size increase from $15.9 \pm 10.6 \mathrm{~nm}$ to $23.2 \pm 11.2 \mathrm{~nm}$, respectively (Figure S3). From the TEM images (Figure S4), we determined a shell thickness of $3.03 \pm 0.96 \mathrm{~nm}(n=36)$ of ND-CP, which corresponds nicely to the diameter of the $C C M V$ capsid. To further assess the structure of the ND-CP, the liquid mode of atomic force microscopy (AFM; Figure S5) was applied. The topographic image of ND-CP showed a narrow distribution with no significant aggregation. The height-sensor images recorded by AFM were converted into deformation images using the NanoScope Analysis 1.8 software to visualize the CP coating. In addition to the height profile images, other nanomechanical properties were simultaneously recorded. In particular, the deformation of the sample caused by the probe was analyzed to obtain indepth information on the structure of the coated NDs. As the ND core is much harder than the CP shell, the deformation of the CP shell under the same stress conditions could be detected with a high intensity. The deformation image revealed clearly that all ND-CP possessed a core-shell structure. 


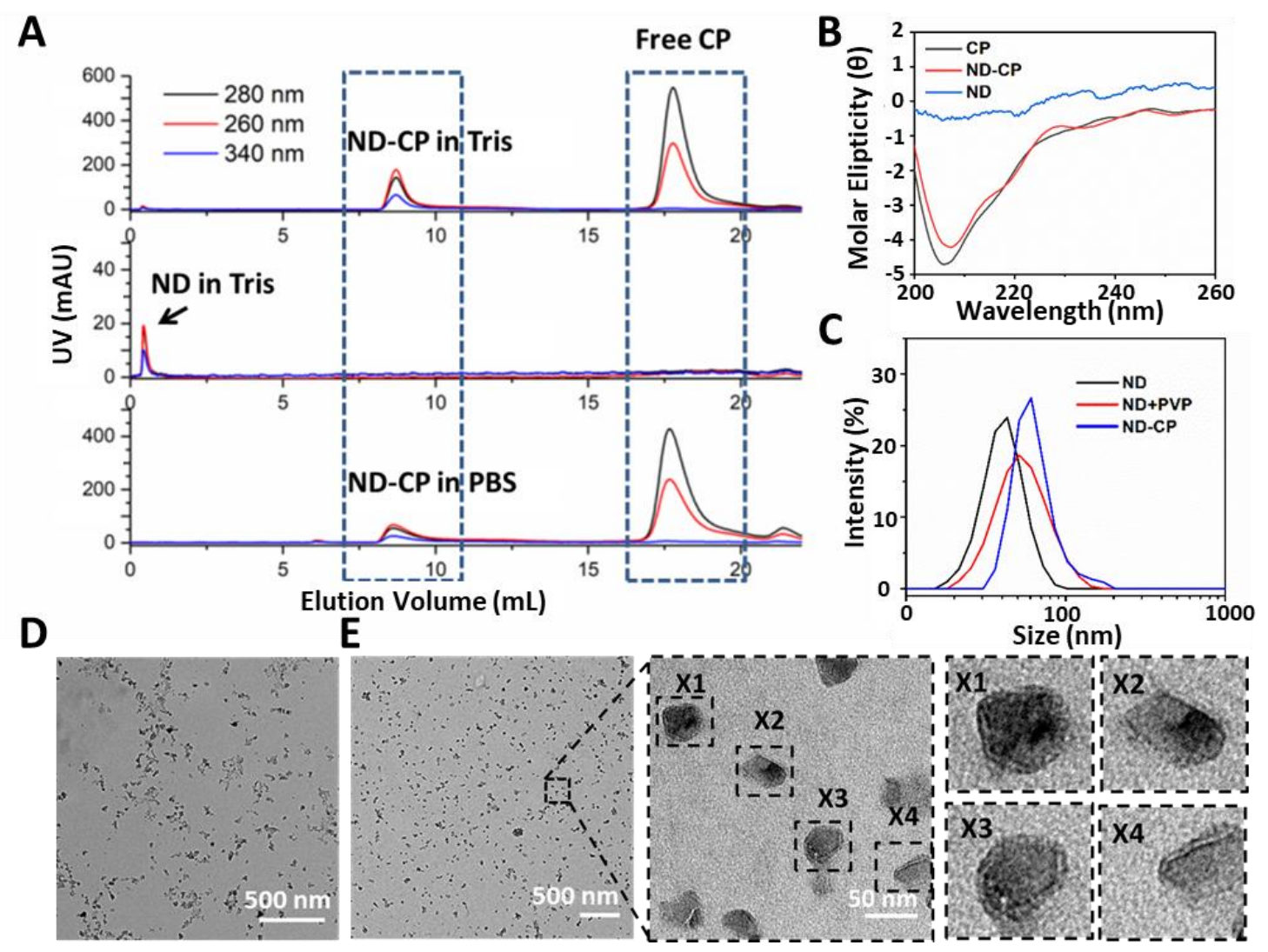

Figure 2. (A) FPLC of ND and ND-CP in Tris buffer (50 mM Tris, $50 \mathrm{mM} \mathrm{NaCl}, 10 \mathrm{mM}$ $\mathrm{KCl}, \mathrm{pH}$ 7.2), ND-CP in PBS buffer (PBS, $150 \mathrm{mM} \mathrm{NaCl}, \mathrm{pH} 7.2)$; (B) CD spectra of ND, CP and ND-CP; (C) DLS diagram of uncoated NDs in water, ND+PVP and ND-CP in Tris buffer; (D) TEM image NDs (scale bar: $500 \mathrm{~nm}$ ); (E) TEM images of ND-CP at different magnifications.

To prove that the optical properties of NDs were retained, the influence of the CP shell on the charge state of the NV centers in NDs was investigated (Figure 3A). Spectra measurements were performed on a custom-built confocal microscope with an excitation 
laser at $532 \mathrm{~nm}$ and $100 \mu \mathrm{W}$ power in front of the objective (oil, NA=1.35) (Figure $\mathrm{S} 1$ ). The spectra of ND-CP revealed that the CP proteins on the surface of NDs did not affect the fluorescence, and the zero phonon lines of $\mathrm{NV}^{-}$centers were clearly visible without any shift or background noise. $\mathrm{NV}^{-}$centers in NDs are very sensitive to the surface states and their charge state can switch to neutral or positive, which is optically nonactive. These results demonstrate that the $\mathrm{CP}$ coating did not affect the charge properties of NV centers. These remained in the optical and spin active state $\left(\mathrm{NV}^{-}\right)$, which is essential for applications of ND-CP in bioimaging, tracking, and nanoscale sensing. The biocompatibility of ND-CP was investigated using HeLa cell line as well as in a chorioallantoic membrane (HET-CAM) ex vivo model ${ }^{42}$. As depicted in Figure 3B, the NDCP showed low cytotoxicity upon treating cells at high concentrations of up to $200 \mu \mathrm{g} / \mathrm{mL}$. It is worth noting that the HET-CAM method represents a potential alternative to animal experiments to assess toxicity and is widely applied to replace animal experiments. ${ }^{42}$ In our HET-CAM assay, we observed hemorrhage from blood vessels that started within 2-4 seconds after applying 1\% sodium dodecyl sulfate (SDS) as positive control. Lysis occurred after 27 seconds, and we observed coagulation within 24 hours. The negative control (phosphate buffered saline) and two concentrations of NG-CP $(50 \mu \mathrm{g} / \mathrm{mL}, 100$ $\mu \mathrm{g} / \mathrm{mL}$ ) did not show any irritation (such as hemorrhage, vascular lysis, or coagulation) within 5 minutes to 24 hours after application (Figure S6). A summary of the results is shown in Table S2. These in ovo results verify the high biocompatibility of ND-CP. 
To track the motion of the ND-CP inside living cells, HeLa lung cancer cells were incubated with $20 \mu \mathrm{g} / \mathrm{mL}$ ND-CP for 4 hours. The measurements were performed on a custom-built confocal microscope with an excitation laser at $532 \mathrm{~nm}$ (Figure S1). As depicted in Figure 3C, ND-CPs were efficiently taken up by Hela cells at concentrations of $20 \mu \mathrm{g} / \mathrm{mL}$. To track ND-CP nanoparticles, the spectrum of the selected spots inside living cells was first measured to confirm the presence of ND-CP in the focus volume (Figure 3D). Then, the fluorescence intensities of the tracked ND-CPs were recorded simultaneously to ensure that the same ND-CP was being tracked (Figure 3F). A representative trajectory of single particles is shown in Figure 3E for 43 minutes and two other trajectories are depicted in Figure S7.

A

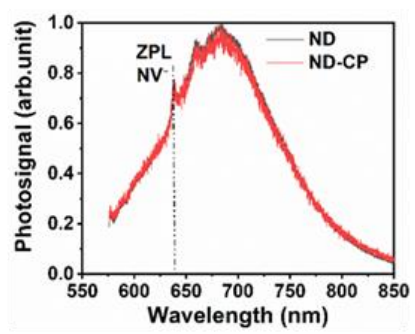

D

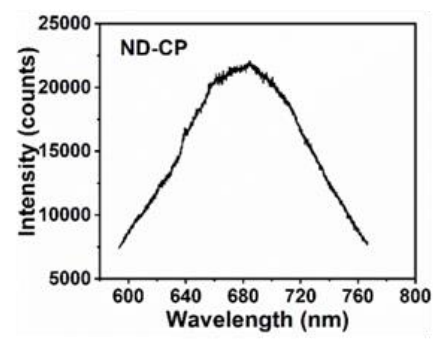

B

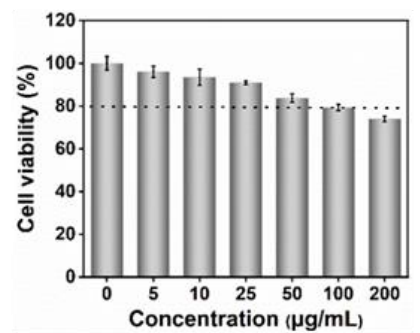

E

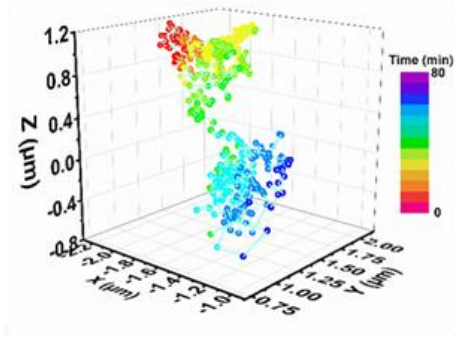

C
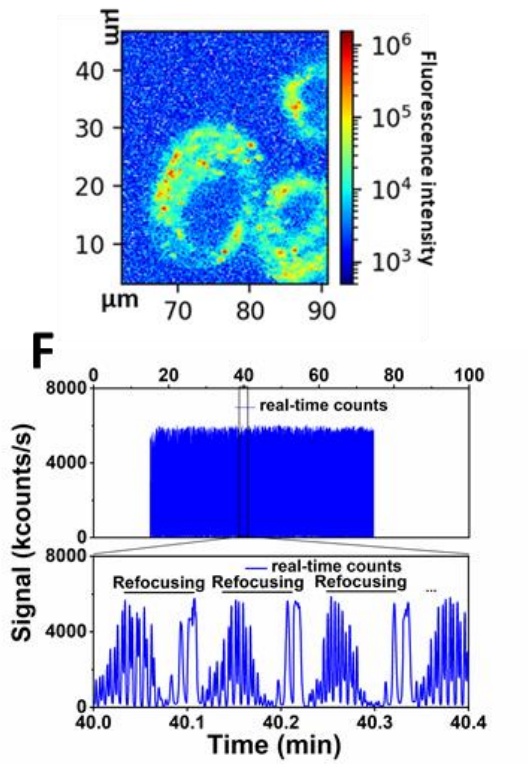

Figure 3. (A) Normalized emission spectra (ex. $532 \mathrm{~nm}$ ) of ND and ND-CP. $\mathrm{NV}^{-}$zero 
phonon lines are visible in both spectra. (B) Cell viability of ND-CP in HeLa cells. (C) X$\mathrm{Y}$ axis confocal microscopy images of ND-CP taken up into HeLa cells at $20 \mu \mathrm{g} / \mathrm{mL}$ for 4 hours incubation. (D) Emission spectra (ex. $532 \mathrm{~nm}$ ) of ND-CP in HeLa cell. (E) The trajectory of tracked ND-CP spot 1 in the intracellular space of HeLa cell. (F) Real-time counts of fluorescence of the tracked ND-CP spot 1 with continuous refocusing (upper panel) and regional enlarged view showing three refocusing steps (lower panel).

The high photostability of NDs provides the opportunity to study intracellular dynamic parameters of ND-CP inside living cells. We performed a set of proof of principle experiments by recording 3D trajectories of three different ND-CP spots. Trajectory 1 was observed for 43 minutes containing 520 points, whereas Trajectories 2 and 3 were recorded for 7 minutes with 88 and 81 points, respectively. For trajectories 2 and 3, long-time trajectory measurements did not succeed due to interference from other ND-CPs that could not be distinguished during the refocusing process. With increasing tracking time, the probability of interference from nearby ND-CPs increases as well, thus limiting the maximum tracking time for individual ND-CPs. The obtained data points allowed calculating the mean square displacement (MSD), which is a measure of the deviation of the position of NP-CP nanoparticles with respect to a reference position over time. The results for each NP-CP spot are presented in Figure 4A (see MSD analysis for calculations details in SI). Subsequently, the diffusion behavior of the ND-CPs was obtained by fitting 
the MSD data with a power-law (Equation S2), where the power index $\alpha$ reflects the diffusion behavior of the tracked nanoparticle with $\alpha<1, \alpha=1$, and $\alpha>1$ to identify the dynamic movements of ND-CPs inside cells. ${ }^{43,} 44$ Within the complex and highly heterogeneous, crowded environment in the cell's cytoplasm, normal diffusion, anomalous diffusion, confined diffusion or directed motion of nanoparticles may occur. ${ }^{45}$ In normal diffusion, nanoparticles diffuse completely unrestricted, whereas directed motion represents an active process that has been observed when small vesicles are transported by molecular machines along microtubules. ${ }^{46,47}$ Confined diffusion has been observed for trapped particles or particles whose free diffusion is confined by cytoskeletal elements. ${ }^{48}$ However, the origin of anomalous diffusion is commonly traced back to the macromolecular crowding in the interior of cells, but its precise nature is still under discussion. $^{45,49}$

According to $\operatorname{Saxton}^{50}$, we focused on data with $t_{\mathrm{lag}}<t_{\text {total }} / 4$, where $\mathrm{t}_{\text {total }}$ is the total time of the trajectory. Our experiments showed a confined diffusion at the short lag time, which was also observed for single-walled-carbon-nanotube (SWNT)-labeled kinesins in COS-7 cells before. ${ }^{51}$ Previously, this behavior was allocated to the existence of mechanical obstacles such as microtubule-microtubule intersections that exist in the complex cytoskeletal network of cells $^{51,52}$. We have distinguished normal and anomalous diffusion of ND-CPs by determining the cumulative distribution function (CDF) of the square 
displacements at a given $t_{\text {lag. }}{ }^{43}, 53$ To provide the CDF computation, the probability distribution function (PDF) of the square displacements was first calculated. The PDF and CDF were analyzed only for trajectory 1 because of the largest number of points, as shown in Figure 4B. The CDF data were fitted with single and double exponential functions, where the double function has fast and slow mobility components. The double fit has a much smaller residual than the single exponential fit. The ratio between the fast and slow mobility parts in the double fit confirms that the diffusion behavior of the ND-CP in HeLa cells is indeed a combination of normal (i.e., MSD $\propto t_{\mathrm{lag}}$ ) and anomalous (i.e., MSD $\propto t_{\mathrm{lag}}{ }^{\alpha}$, with $\alpha$ deviating from one) diffusion. The diffusion coefficient $\left(D_{0}\right)$ calculated from the single exponential fit is equal to $2.09 \times 10^{-4} \mu \mathrm{m}^{2} / \mathrm{s}$. The double exponential fit gives two diffusion coefficients $D_{1}=2.95 \times 10^{-5} \mu \mathrm{m}^{2} / \mathrm{s}$ and $D_{2}=2.93 \times 10^{-4} \mu \mathrm{m}^{2} / \mathrm{s}$. We also calculated the lag-time-dependent diffusion coefficient of the ND-CP in HeLa cell from the MSD data for each of the three recorded trajectories (for more details see SI). We obtained the

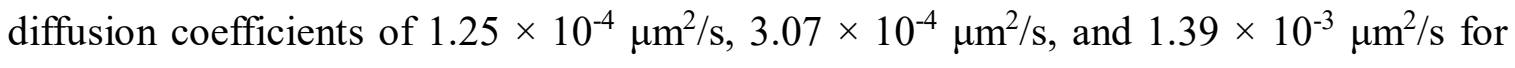
trajectory 1,2, and 3, respectively (Figure 4C). They are comparable to the values obtained from the single and double exponential fits of the CDF data. The nominal diffusion coefficient of the ND-CP in the HeLa cell was then determined to be the average of these three values, that is, $6.07 \times 10^{-4} \mu \mathrm{m}^{2} / \mathrm{s}$.

Interestingly, the recorded intracellular trajectories of ND-CPs are in line with previous 
reports on the diffusion of adeno-associated virions in the cytoplasm of a living HeLa cell, where intracellular trajectories have also been classified as anomalous diffusion and normal diffusion. ${ }^{54}$ It has been speculated that anomalous diffusion occurred due to nonspecific interactions between the virions and obstacles inside the cytoplasm such as organelles, which could also be the origin of the anomalous diffusion of ND-CPs. Therefore, ND-CPs consisting of a fluorescent nanodiamond core and capsid protein corona could represent valuable tools to elucidate the infection pathway of the virions over the cytoplasm.
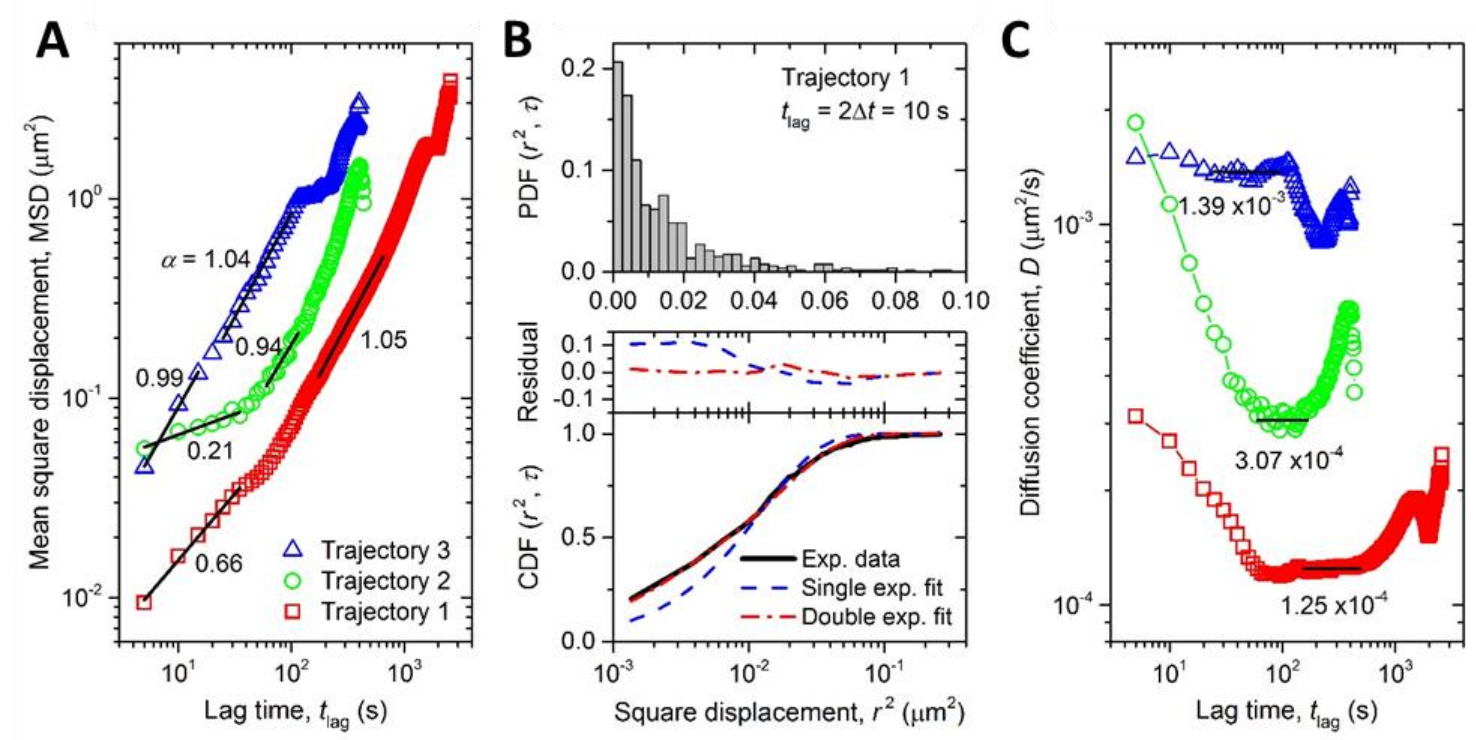

Figure 4. (A) Mean square displacement (MSD) vs. lag time ( $\left.t_{\text {lag }}\right)$ for three independent trajectories. The segments of the MSD profiles at short and intermediate lag times are analyzed using a power law relation. The power indices are indicated. (B) Probability density function (PDF, upper panel, note the linear scale of the $x$ axis) and cumulative density function (CDF, lower panel) distributions of the square displacements at $t_{\text {lag }}=2 \Delta t$ 
$=10 \mathrm{~s}$. The CDF distribution is fitted with single and double exponential functions. The residuals of the fitting are shown on the top of the lower panel. (C) Time-dependent diffusion coefficient $(D)$ of the three trajectories (see (A) for the legends). The nominal $D$ values are calculated as the average values at intermediate lag times, as indicated by the black horizontal lines. In these ranges of the lag times, the power indices in (A) are close to one, implying normal diffusion.

In conclusion, we have developed a virus-inspired NDs hybrid system consisting of a negatively charged fluorescent nanodiamond core stabilized by a corona of virus capsid proteins. ND-CP showed excellent stability in PBS and Tris buffer. Both the NDs and CPs retained their unique features, such as photophysical properties and 3D structure facilitating cellular uptake. Furthermore, they showed good biocompatibility up to 100 $\mu \mathrm{g} / \mathrm{mL}$ in vitro and in vivo, which is essential for bioapplications, such as bioimaging and intracellular trajectory analysis. The estimation of intracellular motions of ND-CP revealed confined diffusion at the beginning, which then changed to normal diffusion (i.e., MSD $\propto$ $t_{\text {lag }}$, which has also been observed for adenovirus-like particles. We believe that ND-CP represent a useful tool to study the intracellular motions over long time periods and with spatiotemporal details in living cells. In combination with optically detected magnetic resonance technique, ${ }^{55}$ one could also envision simultaneous elucidation of intercellular magneto-, electro-, and temperature-information at the nanoscale, which will allow 
acquiring quantitative information on the intracellular pathways of virions. The trajectory analysis of the ND-CP have the potential to enhance the conceptual understanding of cellvirus interactions, host adaption, and immune regulation, and elucidate the interactions between eukaryotes and viruses during infection.

\section{Conflicts of interest}

The authors declare no competing financial interest.

\section{Acknowledgements}

We thank Prof. Dr. Theo Lasser for fruitful scientific discussions, Dr. Siyuan Xiang for the measurement of circular dichroism spectroscopy and Pia Winterwerber for the atomic force microscopy measurement. Y.Wu thanks the China Scholarship Council (CSC) for offering a $\mathrm{PhD}$ scholarship. We are grateful for financial support from the Deutsche Forschungsgemeinschaft (DFG, German Research Foundation) - Project number 316249678 - SFB 1279 (C04). 


\section{Reference}

1. Balasubramanian, G.; Chan, I.; Kolesov, R.; Al-Hmoud, M.; Tisler, J.; Shin, C.; Kim, C.; Wojcik, A.; Hemmer, P. R.; Krueger, A., Nanoscale imaging magnetometry with diamond spins under ambient conditions. Nature 2008, 455 (7213), 648-651.

2. Xiao, J.; Liu, P.; Li, L.; Yang, G., Fluorescence origin of nanodiamonds. The Journal of Physical Chemistry C 2015, 119 (4), 2239-2248.

3. Han, S.; Raabe, M.; Hodgson, L.; Mantell, J.; Verkade, P.; Lasser, T.; Landfester, K.; Weil, T.; Lieberwirth, I., High-contrast imaging of nanodiamonds in cells by energy filtered and correlative lightelectron microscopy: toward a quantitative nanoparticle-cell analysis. Nano Lett. 2019, 19 (3), 2178-2185.

4. Torelli, M. D.; Nunn, N. A.; Shenderova, O. A., A perspective on fluorescent nanodiamond bioimaging. Small 2019, 15 (48), 1902151.

5. Jung, H. S.; Cho, K. J.; Seol, Y.; Takagi, Y.; Dittmore, A.; Roche, P. A.; Neuman, K. C., Polydopamine encapsulation of fluorescent nanodiamonds for biomedical applications. Adv. Funct. Mater. 2018, 28 (33), 1801252.

6. Wu, Y.; Ermakova, A.; Liu, W.; Pramanik, G.; Vu, T. M.; Kurz, A.; McGuinness, L.; Naydenov, B.; Hafner, S.; Reuter, R., Programmable biopolymers for advancing biomedical applications of fluorescent nanodiamonds. Adv. Funct. Mater. 2015, 25 (42), 6576-6585.

7. Harvey, S.; Raabe, M.; Ermakova, A.; Wu, Y.; Zapata, T.; Chen, C.; Lu, H.; Jelezko, F.; Ng, D. Y.; Weil, T., Transferrin - Coated Nanodiamond - Drug Conjugates for Milliwatt Photothermal Applications. Advanced Therapeutics 2019, 2 (11), 1900067.

8. Zhang, Y.; Cui, Z.; Kong, H.; Xia, K.; Pan, L.; Li, J.; Sun, Y.; Shi, J.; Wang, L.; Zhu, Y., One shot immunomodulatory nanodiamond agents for cancer immunotherapy. Adv. Mater. 2016, 28 (14), 2699 2708.

9. Cui, Z.; Zhang, Y.; Xia, K.; Yan, Q.; Kong, H.; Zhang, J.; Zuo, X.; Shi, J.; Wang, L.; Zhu, Y., Nanodiamond autophagy inhibitor allosterically improves the arsenical-based therapy of solid tumors. Nat. Commun. 2018, 9 (1), 1-11.

10. Ryu, T. K.; Baek, S. W.; Kang, R. H.; Choi, S. W., Selective photothermal tumor therapy using nanodiamond - based nanoclusters with folic acid. Adv. Funct. Mater. 2016, 26 (35), 6428-6436.

11. Fu, C.-C.; Lee, H.-Y.; Chen, K.; Lim, T.-S.; Wu, H.-Y.; Lin, P.-K.; Wei, P.-K.; Tsao, P.-H.; Chang, H.-C.; Fann, W., Characterization and application of single fluorescent nanodiamonds as cellular biomarkers. Proceedings of the National Academy of Sciences 2007, 104 (3), 727-732.

12. Chang, Y.-R.; Lee, H.-Y.; Chen, K.; Chang, C.-C.; Tsai, D.-S.; Fu, C.-C.; Lim, T.-S.; Tzeng, Y.-K.; Fang, C.-Y.; Han, C.-C., Mass production and dynamic imaging of fluorescent nanodiamonds. Nat. Nanotechnol. 2008, 3 (5), 284-288.

13. Zhang, B.; Li, Y.; Fang, C. Y.; Chang, C. C.; Chen, C. S.; Chen, Y. Y.; Chang, H. C., Receptor mediated cellular uptake of folate - conjugated fluorescent nanodiamonds: a combined ensemble and single - particle study. Small 2009, 5 (23), 2716-2721.

14. Neumann, P.; Jakobi, I.; Dolde, F.; Burk, C.; Reuter, R.; Waldherr, G.; Honert, J.; Wolf, T.; Brunner, A.; Shim, J. H., High-precision nanoscale temperature sensing using single defects in diamond. Nano Lett. 2013, 13 (6), 2738-2742. 
15. Simpson, D. A.; Morrisroe, E.; McCoey, J. M.; Lombard, A. H.; Mendis, D. C.; Treussart, F.; Hall, L. T.; Petrou, S.; Hollenberg, L. C., Non-neurotoxic nanodiamond probes for intraneuronal temperature mapping. ACS Nano 2017, 11 (12), 12077-12086.

16. Sekiguchi, T.; Sotoma, S.; Harada, Y., Fluorescent nanodiamonds as a robust temperature sensor inside a single cell. Biophysics and physicobiology 2018, 15, 229-234.

17. Ermakova, A.; Pramanik, G.; Cai, J.-M.; Algara-Siller, G.; Kaiser, U.; Weil, T.; Tzeng, Y.-K.; Chang, H.-C.; McGuinness, L.; Plenio, M. B., Detection of a few metallo-protein molecules using color centers in nanodiamonds. Nano Lett. 2013, 13 (7), 3305-3309.

18. Zhang, T.; Liu, G.-Q.; Leong, W.-H.; Liu, C.-F.; Kwok, M.-H.; Ngai, T.; Liu, R.-B.; Li, Q., Hybrid nanodiamond quantum sensors enabled by volume phase transitions of hydrogels. Nat. Commun. 2018, 9 (1), 1-8.

19. Horowitz, V. R.; Alemán, B. J.; Christle, D. J.; Cleland, A. N.; Awschalom, D. D., Electron spin resonance of nitrogen-vacancy centers in optically trapped nanodiamonds. Proceedings of the National Academy of Sciences 2012, 109 (34), 13493-13497.

20. Akiel, R. D.; Zhang, X.; Abeywardana, C.; Stepanov, V.; Qin, P. Z.; Takahashi, S., Investigating functional DNA grafted on nanodiamond surface using site-directed spin labeling and electron paramagnetic resonance spectroscopy. The Journal of Physical Chemistry B 2016, 120 (17), 4003-4008.

21. Xia, K.; Liu, C.-F.; Leong, W.-H.; Kwok, M.-H.; Yang, Z.-Y.; Feng, X.; Liu, R.-B.; Li, Q., Nanometer-precision non-local deformation reconstruction using nanodiamond sensing. Nat. Commun. 2019, 10 (1), 1-9.

22. van der Laan, K.; Hasani, M.; Zheng, T.; Schirhagl, R., Nanodiamonds for in vivo applications. Small 2018, 14 (19), 1703838.

23. Krueger, A.; Lang, D., Functionality is key: recent progress in the surface modification of nanodiamond. Adv. Funct. Mater. 2012, 22 (5), 890-906.

24. Krueger, A., Current issues and challenges in surface chemistry of nanodiamonds. In Nanodiamonds, Elsevier: 2017; pp 183-242.

25. Zhao, L.; Xu, Y.-H.; Akasaka, T.; Abe, S.; Komatsu, N.; Watari, F.; Chen, X., Polyglycerol-coated nanodiamond as a macrophage-evading platform for selective drug delivery in cancer cells. Biomaterials 2014, 35 (20), 5393-5406.

26. Bumb, A.; Sarkar, S. K.; Billington, N.; Brechbiel, M. W.; Neuman, K. C., Silica encapsulation of fluorescent nanodiamonds for colloidal stability and facile surface functionalization. J. Am. Chem. Soc. 2013, 135 (21), 7815-7818.

27. Zhao, L.; Takimoto, T.; Ito, M.; Kitagawa, N.; Kimura, T.; Komatsu, N., Chromatographic separation of highly soluble diamond nanoparticles prepared by polyglycerol grafting. Angew. Chem. Int. Ed. 2011, 50 (6), 1388-1392.

28. Shimkunas, R. A.; Robinson, E.; Lam, R.; Lu, S.; Xu, X.; Zhang, X.-Q.; Huang, H.; Osawa, E.; Ho, D., Nanodiamond-insulin complexes as pH-dependent protein delivery vehicles. Biomaterials 2009, 30 (29), 5720-5728.

29. Tyler, M.; Tumban, E.; Peabody, D. S.; Chackerian, B., The use of hybrid virus - like particles to enhance the immunogenicity of a broadly protective HPV vaccine. Biotechnol. Bioeng. 2014, 111 (12), $2398-$ 2406. 
30. Ruthardt, N.; Lamb, D. C.; Bräuchle, C., Single-particle tracking as a quantitative microscopy-based approach to unravel cell entry mechanisms of viruses and pharmaceutical nanoparticles. Mol. Ther. 2011, 19 (7), 1199-1211.

31. Hema, M.; Vardhan, G. V.; Savithri, H.; Murthy, M., Emerging trends in the development of plant virus-based nanoparticles and their biomedical applications. Recent developments in applied microbiology and biochemistry 2019, 61-82.

32. Douglas, T.; Young, M., Host-guest encapsulation of materials by assembled virus protein cages. Nature 1998, 393 (6681), 152-155.

33. Liu, A.; de Ruiter, M. V.; Zhu, W.; Maassen, S. J.; Yang, L.; Cornelissen, J. J., Compartmentalized Thin Films with Customized Functionality via Interfacial Cross - linking of Protein Cages. Adv. Funct. Mater. 2018, 28 (34), 1801574.

34. Liu, A.; Verwegen, M.; de Ruiter, M. V.; Maassen, S. J.; Traulsen, C. H.-H.; Cornelissen, J. J., Protein cages as containers for gold nanoparticles. The Journal of Physical Chemistry B 2016, 120 (26), $6352-$ 6357.

35. Minten, I. J.; Ma, Y.; Hempenius, M. A.; Vancso, G. J.; Nolte, R. J.; Cornelissen, J. J., CCMV capsid formation induced by a functional negatively charged polymer. Org. Biomol. Chem. 2009, 7 (22), 4685-4688. 36. Schoonen, L.; Nolte, R. J.; van Hest, J. C., Highly efficient enzyme encapsulation in a protein nanocage: towards enzyme catalysis in a cellular nanocompartment mimic. Nanoscale 2016, 8 (30), 14467-14472.

37. Sinn, S.; Yang, L.; Biedermann, F.; Wang, D.; Kübel, C.; Cornelissen, J. J.; De Cola, L., Templated formation of luminescent virus-like particles by tailor-made Pt (II) Amphiphiles. J. Am. Chem. Soc. 2018, 140 (6), 2355-2362.

38. Bancroft, J.; Hiebert, E.; Bracker, C., The effects of various polyanions on shell formation of some spherical viruses. Virology 1969, 39 (4), 924-930.

39. Lavelle, L.; Gingery, M.; Phillips, M.; Gelbart, W.; Knobler, C.; Cadena-Nava, R.; Vega-Acosta,

J.; Pinedo-Torres, L.; Ruiz-Garcia, J., Phase diagram of self-assembled viral capsid protein polymorphs. The Journal of Physical Chemistry B 2009, 113 (12), 3813-3819.

40. Speir, J. A.; Munshi, S.; Wang, G.; Baker, T. S.; Johnson, J. E., Structures of the native and swollen forms of cowpea chlorotic mottle virus determined by X-ray crystallography and cryo-electron microscopy. Structure 1995, 3 (1), 63-78.

41. Douglas, T.; Strable, E.; Willits, D.; Aitouchen, A.; Libera, M.; Young, M., Protein engineering of a viral cage for constrained nanomaterials synthesis. Adv. Mater. 2002, 14 (6), 415-418.

42. Winter, G.; Koch, A. B.; Löffler, J.; Lindén, M.; Solbach, C.; Abaei, A.; Li, H.; Glatting, G.; Beer, A. J.; Rasche, V., Multi-Modal PET and MR Imaging in the Hen's Egg Test-Chorioallantoic Membrane (HET-CAM) Model for Initial in Vivo Testing of Target-Specific Radioligands. Cancers 2020, 12 (5), 1248.

43. Weigel, A. V.; Simon, B.; Tamkun, M. M.; Krapf, D., Ergodic and nonergodic processes coexist in the plasma membrane as observed by single-molecule tracking. Proceedings of the National Academy of Sciences 2011, 108 (16), 6438-6443.

44. Gal, N.; Lechtman-Goldstein, D.; Weihs, D., Particle tracking in living cells: a review of the mean square displacement method and beyond. Rheol. Acta 2013, 52 (5), 425-443.

45. Wagner, T.; Kroll, A.; Haramagatti, C. R.; Lipinski, H.-G.; Wiemann, M., Classification and segmentation of nanoparticle diffusion trajectories in cellular micro environments. PLoS ONE 2017, 12 (1), 
e0170165.

46. Ruan, G.; Agrawal, A.; Marcus, A. I.; Nie, S., Imaging and tracking of tat peptide-conjugated quantum dots in living cells: new insights into nanoparticle uptake, intracellular transport, and vesicle shedding. J. Am. Chem. Soc. 2007, 129 (47), 14759-14766.

47. Bannunah, A. M.; Vllasaliu, D.; Lord, J.; Stolnik, S., Mechanisms of nanoparticle internalization and transport across an intestinal epithelial cell model: effect of size and surface charge. Mol. Pharm. 2014, 11 (12), 4363-4373.

48. Monnier, N.; Guo, S.-M.; Mori, M.; He, J.; Lénárt, P.; Bathe, M., Bayesian approach to MSDbased analysis of particle motion in live cells. Biophys. J. 2012, 103 (3), 616-626.

49. Höfling, F.; Franosch, T., Anomalous transport in the crowded world of biological cells. Reports on Progress in Physics 2013, 76 (4), 046602.

50. Saxton, M. J., Single-particle tracking: the distribution of diffusion coefficients. Biophys. J. 1997, 72 (4), 1744-1753.

51. Fakhri, N.; Wessel, A. D.; Willms, C.; Pasquali, M.; Klopfenstein, D. R.; MacKintosh, F. C.; Schmidt, C. F., High-resolution mapping of intracellular fluctuations using carbon nanotubes. Science 2014, 344 (6187), 1031-1035.

52. Bálint, Š.; Vilanova, I. V.; Álvarez, Á. S.; Lakadamyali, M., Correlative live-cell and superresolution microscopy reveals cargo transport dynamics at microtubule intersections. Proceedings of the National Academy of Sciences 2013, 110 (9), 3375-3380.

53. Schütz, G. J.; Schindler, H.; Schmidt, T., Single-molecule microscopy on model membranes reveals anomalous diffusion. Biophys. J. 1997, 73 (2), 1073-1080.

54. Itto, Y., Diffusion theory for the infection pathway of virus in a living cell. arXiv preprint arXiv:1803.03097 2018.

55. Schirhagl, R.; Chang, K.; Loretz, M.; Degen, C. L., Nitrogen-vacancy centers in diamond: nanoscale sensors for physics and biology. Annu. Rev. Phys. Chem. 2014, 65, 83-105. 